\title{
Determination of the stress-deformed state components of the rock mass around the mine opening fixed by anchors
}

\author{
Alexander Pankratenko ${ }^{1 *}$ and Vadim Bugayev ${ }^{2}$ \\ ${ }^{1}$ National University of Science and Technology MISIS (Moscow Institute of Steel and Alloys), \\ 119991, Leninskij Av., 4, Moskow, Russia \\ ${ }^{2}$ VostokCoal Management Company, 143084, estate 100, "Residence on Rublevka" Business Center, \\ Usovo, Odinstovsky District, Moscow Region, Russia
}

\begin{abstract}
The analytical investigation of the stress-deformed state of the rock mass around mine with random cross-section and anchorage is completed. The problem is solved in reliance on tangential stress, that arising in anchors.
\end{abstract}

\section{Introduction}

Some theoretical aspects of calculating the joint usage of anchors and concrete support remain undeveloped in the frame of underground structures. [1-2].

For determination the stress-deformed state of the rock mass around mine, that fixed by the frame of anchors, it is necessary to use the elasticity theory of stress distribution around open hole and elasticity theory of stresses in elastic range, which is relaxed by open hole [35].

\section{Method of calculation}

Expression for components of stress in rock mass, that fixed by anchors [1]

$$
\begin{aligned}
& \sigma_{r}=\sigma_{r}^{(0)}+\sigma_{r}^{(1)}+\sigma_{r}^{(\Sigma)}, \\
& \sigma_{\theta}=\sigma_{\theta}^{(0)}+\sigma_{\theta}^{(1)}+\sigma_{\theta}^{(\Sigma)}, \\
& \tau_{r \theta}=\tau_{r \theta}^{(0)}+\tau_{r \theta}^{(1)}+\tau_{r \theta}^{(\Sigma)},
\end{aligned}
$$

Where, $\sigma_{r}, \sigma_{\theta}, \tau_{r \theta}$ - total stresses; $\sigma_{r}^{(0)}, \sigma_{\theta}^{(0)}, \tau_{r \theta}^{(0)}$ - inherent stresses of rock mass; $\sigma_{r}^{(1)}, \sigma_{\theta}^{(1)}, \tau_{r \theta}^{(1)}$ - secondary stresses induced by mine excavation; $\sigma_{r}^{(\Sigma)}, \sigma_{\theta}^{(\Sigma)}, \tau_{r \theta}^{(\Sigma)}$ stress combination from effect of concentration forces.

Components of inherent and secondary stresses for different forms of cross-section can be find in research [3].

\footnotetext{
*Corresponding author: pankrat54@bk.ru
} 
For determination of stress combination $\sigma_{r}^{(\Sigma)}, \sigma_{\theta}^{(\Sigma)}, \tau_{r \theta}^{(\Sigma)}$ it is necessary to solve the problem of elasticity theory of effect of concentration forces in elastic plane, that relaxed by arbitrarily-shaped hole. On a random point $z_{0}$ that has coordinates $\left(r_{0}, \theta_{0}\right)$, on the angle $\theta_{1}$ exert a concentrated force $Q$. When force direction $Q$ is inconsistent with normal to mine circuit, there forming the tangential stresses. It must be considered on practice. From the elasticity theory [3] we know functions of complex potentials $\varphi$ and $\psi$, that describe stress-deformed state of infinite plane from effect of concentration forces. In this case, this potentials take the form

$$
\begin{aligned}
& \varphi(z)=-\frac{X+i Y}{2 \pi(\chi+1)} \ln \left(z-z_{0}\right)+\varphi_{0}(z), \\
& \psi(z)=\chi \frac{X+i Y}{2 \pi(\chi+1)} \ln \left(z-z_{0}\right)+\frac{X+i Y}{2 \pi(\chi+1)} \cdot \frac{z_{0}}{z-z_{0}}+\psi_{0}(z),
\end{aligned}
$$

Where, $X, Y$ - projections of force $Q$ on real axis and conjugate axis; $\chi=3-4 v, v$ Poisson ratio. This function submits in a form:

$$
z=\omega(\xi)=c_{0} \xi+\frac{c_{1}}{\xi}+\frac{c_{2}}{\xi^{2}}+\ldots
$$

For the new variable, the expression (2) takes on form:

$$
\begin{aligned}
& \varphi(\xi)=-\frac{Q \sigma_{1}}{2 \pi(\chi+1)} \ln \left(\xi-\xi_{0}\right)+\varphi_{0}(\xi), \\
& \psi(\xi)=\chi \frac{Q \sigma_{1}^{-1}}{2 \pi(\chi+1)} \ln \left(\xi-\xi_{0}\right)+\frac{\overline{\omega(\xi)}}{\omega^{\prime}(\xi)} \frac{Q \sigma_{1}}{2 \pi(\chi+1)} \cdot \frac{1}{\xi-\xi_{0}}+\psi_{0}(\xi),
\end{aligned}
$$

Where, $\varphi_{0}(\xi), \psi_{0}(\xi)$ - holomorphic functions of the off-line unit circle, that includes point at infinity.; $\sigma_{1}=\exp \left(i \theta_{1}\right)$.

Boundary conditions take on form

$$
\begin{aligned}
& \varphi(\sigma)+\frac{\omega(\sigma)}{\omega^{\prime}(\sigma)} \varphi^{\prime}(\sigma)+\psi(\sigma)=0, \\
& \psi(\sigma)+\frac{\overline{\omega(\sigma)}}{\omega^{\prime}(\sigma)} \varphi^{\prime}(\sigma)+\psi(\sigma)=0,
\end{aligned}
$$

here $\sigma$ - the point of unit circle $\sigma=\exp (i \theta)$.

Substitute $\varphi(\sigma)$ and $\psi(\sigma)$, computed form (4), deduce

$$
\begin{aligned}
& \varphi_{0}(\sigma)+\frac{\omega(\sigma)}{\overline{\omega^{\prime}(\sigma)}} \overline{\varphi_{0}^{\prime}(\sigma)}+\overline{\psi_{0}(\sigma)}=f(\sigma), \\
& \psi_{0}(\sigma)+\frac{\overline{\omega(\sigma)}}{\omega^{\prime}(\sigma)} \varphi_{0}^{\prime}(\sigma)+\psi_{0}(\sigma)=\overline{f(\sigma),}
\end{aligned}
$$

here

$$
\begin{aligned}
f(\sigma) & =\frac{Q \sigma_{1}}{2 \pi(\chi+1)}\left[\ln \left(\sigma-\xi_{0}\right)-\chi \ln \left(\sigma^{-1}-\xi_{0}\right)\right]+ \\
& +\frac{Q \sigma_{1}^{-1}}{2 \pi(\chi+1)} \frac{\sigma}{1-\sigma \xi_{0}} \frac{1}{\omega^{\prime}(\sigma)}\left[\omega(\sigma)-\omega\left(\xi_{0}\right)\right] .
\end{aligned}
$$


Multiply both member if first inequation (6) by Cauchy kernel $\frac{1}{2 \pi i} \frac{d \sigma}{\sigma-\xi}$ and then, integrate over unit circle circuit $\mathrm{G}$, account point $\xi$, that arranged outward $\mathrm{G}$ (counterclockwise). Since $\varphi_{0}(\xi)$ is holomorphis outward G, and functions $\frac{\omega(\xi)}{\omega^{\prime}(\xi)} \overline{\varphi_{0}^{\prime}}(\xi)$ and $\overline{\psi_{0}(\xi)}$ - in G, then

$$
\begin{aligned}
& \frac{1}{2 \pi i} \int \frac{\varphi_{0}(\xi) d \sigma}{\sigma-\xi}=-\varphi_{0}(\xi), \\
& \frac{1}{2 \pi i} \int \frac{\omega(\xi)}{\overline{\omega^{\prime}(\xi)} \varphi_{0}^{\prime}(\xi) \frac{d \sigma}{\sigma-\xi}=0,} \\
& \frac{1}{2 \pi i} \int \frac{\psi_{0}(\xi) d \sigma}{\sigma-\xi}=0 .
\end{aligned}
$$

It follows that

$$
\varphi_{0}(\xi)=-\frac{1}{2 \pi i} \int \frac{f(\sigma) d \sigma}{\sigma-\xi}
$$

Submit function

$$
g(\sigma)=\frac{\sigma}{1-\sigma \overline{\xi_{0}}} \frac{1}{\omega^{\prime}(\sigma)}\left[\omega(\sigma)-\omega\left(\xi_{0}\right)\right]
$$

In a form of Laurent expansion

$$
g(\sigma)=\sum_{k=1}^{\infty} A_{k} \sigma^{k}+\sum_{k=0}^{\infty} A_{-k} \sigma^{-k}
$$

Then, after integrating in (8) deduce

$$
\varphi_{0}(\xi)=\frac{\chi Q \sigma_{1}}{2 \pi(\chi+1)} \ln \frac{\xi_{0} \cdot \xi}{\xi_{0} \cdot \xi-1}+\frac{Q \sigma_{1}^{-1}}{2 \pi(\chi+1)} \sum_{k=1}^{\infty} A_{-k} \xi^{-k}
$$

Apply to second equation (6). Perform similar operations, will receive

$$
\psi_{0}(\xi)=\frac{Q \sigma_{1}^{-1}}{2 \pi(\chi+1)} \ln \frac{\xi_{0} \cdot \xi-1}{\xi_{0} \cdot \xi}+\frac{Q \sigma_{1}}{2 \pi(\chi+1)} \sum_{k=1}^{\infty} \overline{A_{k}} \xi^{-k}-\frac{\overline{\omega(\xi)}}{\omega^{\prime}(\xi)} \cdot \varphi_{0}^{\prime}(\xi)
$$

Evaluate expansion coefficients $A_{ \pm k}$ for the random cross-section of mine. Substitute $\omega(\sigma)$, that evaluated from (3), in (9) and set equal to (10) coefficients at equal of $\sigma$, deduce

$$
\left\{\begin{array}{l}
A_{k}=\omega\left(\xi_{0}\right) \sum_{j=0}^{\infty} \frac{T_{j+k}}{\xi_{0}^{j+1}}-c_{0} \sum_{j=0}^{\infty} \frac{T_{j+k-1}}{\xi_{0}^{j+1}}-\sum_{i=0}^{\infty} c_{i} \sum_{j=0}^{\infty} \frac{T_{j+k+i}}{\xi_{0}^{j+1}}, \quad k \geq 1, \\
A_{-k}=\omega\left(\xi_{0}\right) \sum_{j=0}^{\infty} \frac{T_{j}}{\xi_{0}^{j+k+1}}-c_{0} \sum_{j=0}^{\infty} \frac{T_{j}}{\xi_{0}^{j+k+2}}-\sum_{i=0}^{\infty} c_{i} \sum_{j=0}^{\infty} \frac{T_{j}}{\xi_{0}^{j+k+1-i}}-\sum_{i=0}^{\infty} c_{i} \sum_{j=0}^{\infty} \frac{T_{j+i}}{\xi_{0}^{j+1}}, \quad k \leq 0,
\end{array}\right.
$$

here $T_{j}$ - the expansion coefficients into a series Taylor from function $\frac{1}{\omega^{\prime}(\sigma)}$.

Components of stress are expressed be means of complex potentials KolosofMuskhelishvili 


$$
\begin{aligned}
& \sigma_{r}^{(\Sigma)}+\sigma_{\theta}^{(\Sigma)}=4 \operatorname{Re} \frac{\varphi^{\prime}(\xi)}{\omega^{\prime}(\xi)}, \\
& \sigma_{\theta}^{(\Sigma)}-\sigma_{r}^{(\Sigma)}+2 i \tau_{r \theta}^{(\Sigma)}=\frac{2 \xi^{2}}{r^{2} \overline{\omega^{\prime}(\xi)}}\left(\overline{\omega(\xi)} \frac{\varphi^{\prime \prime}(\xi) \omega^{\prime}(\xi)-\varphi^{\prime}(\xi) \omega^{\prime \prime}(\xi)}{\omega^{\prime 2}(\xi)}+\psi^{\prime}(\xi)\right) \text {. }
\end{aligned}
$$

\section{Conclusion}

Hence, stress condition of the rock mass around mine with random cross-section and fixed by anchors with provision for tangential stress specify lit-by-lit movement is evaluated.

\section{References}

1. A. Prokopov, V. Matua, V. Akopyan, Procedia Engineering, 150 (2016)

2. M. Pleshko, B. Meskhi, B., M. Pleshko, MATEC Web of Conferences, 170, 03023 (2018)

3. A. Pankratenko, M. Pleshko and A. Isaev, MATEC Web of Conferences, 7, 02026 (2018)

4. F.I. Yagodkin, A.Y. Prokopov, M.S. Pleshko, A.N. Pankratenko, IOP Conference Series: Earth and Environmental Science, 87(6), 062014 (2017)

5. F. Yagodkin, M. Pleshko, A. Prokopov, Procedia Engineering, 206 293-298 (2017) 